\title{
Estructura factorial, fiabilidad y validez de la escala de adicción al trabajo (WorkBAT): Versión española
}

\author{
Joan Boada-Grau ${ }^{1}$, Aldo-Javier Prizmic-Kuzmica ${ }^{2}$, María-José Serrano-Fernández ${ }^{1}$ \\ y Andreu Vigil-Colet ${ }^{1}$ \\ 1 Universitat Rovira i Virgili (Tarragona, Spain) \\ 2 Escuela de Alta Dirección y Administración (Barcelona, Spain)
}

\begin{abstract}
Resumen: El objetivo de este estudio fue realizar la adaptación al españo del inventario WorkBAT de Spence y Robbins (1992) y analizar sus propiedades psicométricas. Para ello se administró una versión adaptada al español a dos muestras, la primera de ellas de 285 empleados $(56.5 \%$ mujeres y $43.5 \%$ hombres) y la segunda de 342 empleados $(52.9 \%$ mujeres y $47.1 \%$ hombres). A la primera muestra, se aplicó el análisis factorial exploratorio extrayéndose dos subescalas: motivación/implicación y disfute en el trabajo. A la segunda muestra, se aplicó el análisis factorial confirmatorio (ESEM) replicando los resultados anteriores. Ambas subscalas mostraron una buena fiabilidad. Las puntuaciones obtenidas en las mismas fueron relacionadas con diversos correlatos externos y otras escalas mostrando una buena validez convergente y criterial. Los resultados indican que la presente escala puede utilizarse para evaluar la adicción al trabajo y, además, es un instrumento con una apreciable bondad psicométrica. Futuras investigaciones podrían utilizar el WorkBAT como una herramienta de screening en combinación con otros instrumentos.

Palabras Clave: Adicción al trabajo; WorkBAT (Spence y Robbins); escala; adaptación español; estudio instrumental.
\end{abstract}

\section{Introducción}

El fenómeno de la adicción al trabajo empieza a ser global. Actualmente la investigación no se limita a los Estados Unidos con sus estudios pioneros sobre el tema (Burke, $1999 \mathrm{y}$ 2001; McMillan, Brady, O'Driscoll y Marsh, 2002; Porter, 1996; Robinson, 1996, 1998, 2000; Spence y Robbins, 1992), también se han realizado investigaciones en otros continentes como Asia (Ersoy-Kart, 2005; Huang, Hu, y Wu, 2010; Kanai y Wakabayashi, 2001; Kanai, Wakabayashi, y Fling, 1996), Europa (Andreassen, Hetland y Pallesen, 2010; Andreassen, Ursin, y Eriksen, 2007; Del-Líbano, Llorens, Salanova, y Schaufeli, 2010; Schaufeli, Shimazu, y Taris, 2009; Schaufeli, Taris, y Rhenen, 2008), y en Oceanía (McMillan et al., 2002).

El constructo científico adicción al trabajo (workaholism) lo acuñó por primera vez Oates $(1968,1971)$. Este autor lo definió como la necesidad incontrolable de trabajar de forma constante que puede tener una persona. En realidad, la adicción al trabajo es la etiqueta común que se utiliza para describir un foco atípico sobre el trabajo (McMillan, O'Driscoll, Marsh y Brady, 2001; Poelmans y Masuda, 2009). Una persona adicta al trabajo presenta varios patrones de conducta característicos; en primer lugar, pasa una cantidad significativa de tiempo en el trabajo y con las actividades intrínsecas

* Dirección para correspondencia [Correspondence address]: Joan Boada-Grau. Universitat Rovira i Virgili. Centre de Recerca d'Avaluació i Mesura de la Conducta (CRAMC). Carretera de Valls, s/n. 43007 Tarragona (España). E-mail: joan.boada@urv.cat

\begin{abstract}
Title: Factorial structure, reliability and validity of the workaholism scale (WorkBAT): Spanish version.

Abstract: This study aims to carry out an adaptation into Spanish of Spence and Robbins' WorkBAT inventory (1992) and to analyse its psychometric properties. With this objective in mind a version adapted into Spanish of two samples, the first consisting of 285 employees $(56.5 \%$ women and $43.5 \%$ men) and the second of 342 employees (52.9\% women and $47.1 \% \mathrm{men})$. The first sample, after it was subjected to an exploratory factor analysis, yielded two subscales: motivation/commitment and enjoyment at work. Confirmatory factor analysis (ESEM) was then applied to the second sample, replicating the previous results. Both subscales showed good reliability. The scores from the scales were then related to various external correlates and to other scales and displayed good convergent and criterion validity. The findings indicate that the present scale can be used to evaluate workaholism and furthermore that it is fundamentally a psychometrically sound instrument. The WorkBAT can be used in future research studies as a screening tool in combination with other instruments.

Key words: Workaholism; WorkBAT (Spence y Robbins); scale; spanish adaptation; instrumental study.
\end{abstract}

que conlleva el mismo (Spence y Robbins, 1992), produciendo consecuencias negativas en su desarrollo social, familiar y de ocio (Scott, Moore y Miceli, 1997), por ejemplo, Ersoy-Kart (2005) considera que las personas adictas trabajan al menos cincuenta horas a la semana. Segundo, tiene muchas expectativas en su trabajo más allá de los requerimientos del mismo y de las propias necesidades económicas (Scott et al., 1997). Tercero, su actitud ante el trabajo se concreta en dedicar más energía al trabajo de lo que es estrictamente necesaria (Andreassen et al., 2007); y cuarto, la persistencia y la frecuencia que tiene en su trabajo hace que piense en el trabajo aunque no esté en él (Scott et al., 1997).

En este marco, Spence y Robbins (1992) consideran que el workaholism implica estar altamente involucrado en el trabajo, tener la sensación de estar impulsado a trabajar por presiones internas y bajo disfrute en el trabajo. Estos dos autores, usando una metodología deductiva (McMillan et al., 2002) han generado un modelo teórico de workaholism que es tridimensional basado en el Work Involvement (en adelante, WI), el Drive (en adelante, D) y el Work Enjoyment (en adelante, J). El WI indica una actitud generalizada de implicación con el trabajo, el $D$ evalúa una presión interna para trabajar y que, además, sea mantenida por presiones externas, y el $J$ expresa el disfrute y la satisfacción en el trabajo (McMillan et al., 2002; Spence y Robbins, 1992).

El presente modelo ha generado un instrumento denominado WorkBAT (25 ítems) el cual se ha desarrollado y validado utilizando distintas muestras de EE.UU. (Robinson, 1996; Spence y Robbins, 1992) y Canadá (Burke, 1999, 2001); también se ha utilizado en Japón (Kanai et al., 1996), 
Nueva Zelanda (McMillan, et al., 2002), Turquía (Ersoy-Kart, 2005), China (Huang et al., 2010) y Noruega (Andreassen et al., 2010; Burke, Richardsen y Martinussen, 2002). Es necesario destacar que los resultados son contradictorios, en las distintas versiones, dado que no todos replican la estructura trifactorial de Spence y Robbins (1992). Así, algunas adaptaciones se configuran bien en dos factores (Kanai et al., 1996; McMillan et al., 2002; Ersoy-Kart, 2005) o en cinco factores (Huang et al., 2010). En cuanto a las muestras utilizadas en las diversas adaptaciones, estas son muy diversas (por ejemplo, empleados, estudiantes universitarios sin graduar y estudiantes de MBA) y, además, los análisis factoriales exploratorios (en adelante, AFE) son distintos (rotación varimax y método de ejes principales) y, sólo, dos investigaciones (Andreassen et al., 2010; Ersoy-Kart, 2005) utilizan el análisis factorial confirmatorio (en adelante, AFC).

A partir de las investigaciones realizadas por Spence y Robbins (1992), formulamos tres hipótesis que son las siguientes:

Hipótesis 1: La consistencia interna evaluada a partir del AFE distinguirá los tres componentes (WI, $D$ y $J$ ) de la escala original inglesa.

Hipótesis 2: El AFC replicará los factores extraídos del AFE.

Hipótesis 3: Los factores de la escala tendrán una fiabilidad adecuada.

La adicción al trabajo, medida a través del WorkBAT, se ha correlacionado con la depresión (Haymon, 1992), la ansiedad (Haymon, 1992; Robinson, 1996), el enojo (Haymon, 1992), el estrés laboral (Aziz y Zickar, 2006; Jackson, 1992; Kanai et al., 1996; Spence y Robbins, 1992), el engagement (Andreassen et al., 2007), las psicosomatizaciones (Andreassen et al., 2007; Kanai et al., 1996; Spence y Robbins, 1992), los transtornos gatrointestinales y músculo esqueléticos (Andreassen et al., 2007), los conflictos trabajo-familia (Bakker, Demerouti, y Burke, 2009) y trabajo-vida (Bonebright, Clay y Ankenmann, 2000), la satisfacción laboral (Huang et al., 2010), la motivación laboral intrínseca (McMillan et al., 2002), la satisfacción con el trabajo (Huang et al., 2010; McMillan et al., 2002), y la no delegación (Burke, 1999; Kanai et al., 1996; Spence y Robbins, 1992).

En la presente investigación se han utilizado un conjunto de variables para mostrar la validez convergente de la escala adaptada a la lengua española. Por un lado, se han utilizado correlatos externos para determinar la validez del WorkBAT como la edad (Johnstone y Johnston, 2005), la antigüedad (en el lugar de trabajo y en la empresa) y las horas trabajadas durante la semana (Andreassen et al., 2007; Aziz y Zickar, 2006; Burke y Ng, 2007; Huang et al., 2010; Johnstone y Johnston, 2005; McMillan et al., 2002; Porter, 1996). Además, se han tenido en cuenta un conjunto de correlatos externos no utilizados en las otras investigaciones, entre otros, la "frecuencia con que uno se lleva trabajo a casa", las "noches que uno se había despertado pensando en temas de trabajo", las "citas personales demoradas", las "llamadas te- lefónicas en fin de semana", las "horas extras trabajadas en el último año" y "los días de vacaciones disfrutados".

Por otro lado, se han considerado cinco constructos como indicios de validez. El primero, la irritación (Merino, Carbonero, Moreno y Morante, 2006) es un término acuñado en las investigaciones iniciadas en la Universidad de Leipzig (Mohr, 1986). La irritación hace referencia a un estado de agotamiento psicológico progresivo que no puede paliarse con los descansos normales. Así, la irritación puede surgir cuando la persona experimenta una discrepancia entre una situación dada y la consecución de un objetivo personal importante.

Segundo, el burnout ha sido descrito como un síndrome que tiene tres componentes: el agotamiento emocional, el cinismo y la baja autoeficacia profesional (Maslach, Schaufeli y Leiter, 2001). Este síndrome se desarrolla, especialmente, en las profesiones que asisten a personas (Maslach, 1982). Algunas investigaciones han sugerido que los componentes del workaholism tienen relación con el burnout (Andreassen et al., 2007; Huang et al., 2010).

En tercer lugar, la adicción al trabajo es considerada como el reflejo de algunos rasgos de personalidad (Burke, Matthiesen y Pallesen, 2006; Ng, Sorensen y Feldman, 2007). La teoría de los cinco factores (Vigil-Colet, Morales-Vives, Camps, Tous y Lorenzo-Seva, en prensa) indica que la personalidad está configurada por la extraversión (por ejemplo, positivo, enérgico y alegre), la responsabilidad, (por ejemplo, autodisciplinado y ordenado), la estabilidad emocional, (por ejemplo, seguro), la amabilidad (por ejemplo, amistoso y cooperativo) y la apertura a la experiencia (por ejemplo, abierto a nuevas ideas y el cambio). Autores como Andreassen et al. (2010) y Burke et al (2006) han hallado ciertas relaciones entre los rasgos de personalidad y el workaholism.

Cuarto, en el marco de la creencias obsesivas (Belloch, Cabedo, Morillo, Lucero y Carrió, 2003) hay dos dimensiones importantes a tener en cuenta. Una de ellas, es la responsabilidad excesiva que alude a la creencia de que uno puede provocar y, por tanto, debe prevenir acontecimientos negativos importantes, lo que conlleva que la persona se sienta muy responsable de todo lo que ocurre a su alrededor. También incluye sentirse igual de responsable por errores de comisión que de omisión y considerar que si uno tiene parte de responsabilidad en algo, es lo mismo que tenerla por completo. La otra, se refiere al perfeccionismo. Éste consiste en creer que hay una solución perfecta para cada problema, y que, por tanto, hacerlo perfecto es posible y necesario, de modo que cualquier fallo tendrá consecuencias graves. Se han hallado correlaciones positivas entre el workaholism y el perfeccionismo (Burke, 1999; Spence y Robbins, 1992).

Finalmente, se ha cosiderado también la impulsividad. Dickman (1990) distingue dos formas independientes de impulsividad. Una es funcional, ésta se define como la tendencia a tomar decisiones en función de las demandas externas con la finalidad de recibir alguna recompensa. El otro tipo de impulsividad es la disfuncional, que indica la rapidez y la no reflexión en la toma de decisiones, y que conlleva con- 
secuencias negativas para la persona. Robinson (1996) encontró que la impaciencia como una característica de la impulsividad (Whiteside y Lynam, 2001) correlaciona con el workaholism.

Al hilo de lo anterior, teniendo en cuenta las investigaciones precedentes, consideramos otra hipótesis:

Hipótesis 4: Los factores de la escala correlacionarán con otras variables (socio-demográficas, correlatos externos, irritación, burnout, personalidad e impulsividad) aportando indicios de validez convergente de la escala investigada.

\section{Método}

\section{Participantes}

En el presente estudio han participado trabajadores por cuenta ajena, que están en activo en la actualidad. Son residentes en España (Comunidad Autónoma de Catalunya). Se han utilizado dos muestras cuyas características se describen en la Tabla 1.

Tabla 1. Características sociodemográficas de los participantes de las dos muestras.

\begin{tabular}{|c|c|c|c|}
\hline & & $\begin{array}{l}\text { Muestra } 1 \\
(\mathrm{~N}=285)\end{array}$ & $\begin{array}{l}\text { Muestra } 2 \\
(\mathrm{~N}=342)\end{array}$ \\
\hline \multirow{2}{*}{ Género } & Hombres & $43.5 \%$ & $47.1 \%$ \\
\hline & Mujeres & $56.5 \%$ & $52.9 \%$ \\
\hline Edad (años) & & $\mathrm{M}=43.31(\mathrm{DT}=9.46)$ & $\mathrm{M}=45.93(\mathrm{DT}=9.61)$ \\
\hline \multirow[t]{5}{*}{ Estado Civil } & Casados & $68.9 \%$ & $66.7 \%$ \\
\hline & Divorciado o separado & $15.0 \%$ & $8.3 \%$ \\
\hline & Pareja de hecho & $7.8 \%$ & $14.0 \%$ \\
\hline & Soltero & $7.3 \%$ & $10.3 \%$ \\
\hline & Viudo & $1.0 \%$ & $0.7 \%$ \\
\hline Hijos & Número & $\mathrm{M}=1.62(\mathrm{DT}=1.11)$ & $\mathrm{M}=1.71(\mathrm{DT}=1.22)$ \\
\hline \multirow[t]{3}{*}{ Antigüedad (años) } & En su trabajo actual & $\mathrm{M}=12.11(\mathrm{DT}=10.5)$ & $\mathrm{M}=10.08(\mathrm{DT}=9.72)$ \\
\hline & En su profesión & $\mathrm{M}=18.47(\mathrm{DT}=15.54)$ & $\mathrm{M}=16.34(\mathrm{DT}=16.24)$ \\
\hline & En su empresa actual & $\mathrm{M}=15.04(\mathrm{DT}=11.01)$ & $\mathrm{M}=12.28(\mathrm{DT}=12.10)$ \\
\hline \multirow[t]{6}{*}{ Nivel Formativo } & Estudios primarios acabados & $23.7 \%$ & $1.7 \%$ \\
\hline & Estudios secundarios acabados & $40.2 \%$ & $28.5 \%$ \\
\hline & Diplomatura o ingeniero técnico & $14.9 \%$ & $40.7 \%$ \\
\hline & Licenciado, ing. superior o arquitecto & $12.9 \%$ & $13.5 \%$ \\
\hline & Máster y/o doctorado & $6.7 \%$ & $8.7 \%$ \\
\hline & Ningún título académico & $1.6 \%$ & $6.9 \%$ \\
\hline Sectores & Mediación financiera & $14.7 \%$ & $11.4 \%$ \\
\hline \multirow[t]{17}{*}{ Productivos } & Educación y servicios sociales & $10.6 \%$ & $10.1 \%$ \\
\hline & Sanidad y hospitales & $10.5 \%$ & $11.7 \%$ \\
\hline & Comercio & $7.5 \%$ & $6.1 \%$ \\
\hline & Telecomunicaciones & $7.1 \%$ & $2.2 \%$ \\
\hline & Metalúrgico y afines & $6.9 \%$ & $4.2 \%$ \\
\hline & Administración pública & $6.3 \%$ & $5.5 \%$ \\
\hline & Farmacéutico & $5.9 \%$ & $4.2 \%$ \\
\hline & Químico & $5.4 \%$ & $8.8 \%$ \\
\hline & Seguridad & $3.8 \%$ & $3.4 \%$ \\
\hline & Servicios destinados a ventas & $3.6 \%$ & $2.9 \%$ \\
\hline & Informática & $3.5 \%$ & $3.1 \%$ \\
\hline & Consultoría en general & $3.3 \%$ & $5.1 \%$ \\
\hline & Hostelería & $2.8 \%$ & $5.4 \%$ \\
\hline & Distribución & $2.5 \%$ & $3.2 \%$ \\
\hline & Turismo & $2.4 \%$ & $6.5 \%$ \\
\hline & Alimentación & $2.1 \%$ & $4.1 \%$ \\
\hline & Otros & $1.1 \%$ & $2.1 \%$ \\
\hline
\end{tabular}

\section{Instrumentos}

La escala de Adicción al Trabajo (WorkBAT; Burke, 1999, 2001; Burke et al., 2002; McMillan et al., 2002; Spence y Robbins, 1992) tiene 25 ítems en su versión original en inglés. La validación del instrumento se realizó llevando a cabo los pasos que se señalan en la literatura científica sobre la adaptación de instrumentos de evaluación (Brislin, 1970;
Hambleton, 1994; Hambleton, Merenda y Spielberger, 2005; Muñiz y Bartram, 2007): Traducción por expertos de los ítems al español, grupo de discusión sobre la traducción realizada de los ítems y retrotraducción de nuevo al inglés ("back-translation") y comprobación de la equivalencia entre ambas versiones. El formato de respuesta es de cinco puntos (De 1= Nada de acuerdo a 5= Totalmente de acuerdo). Inicialmente la versión inglesa tiene tres factores: WI tiene 8 ítems 
(Fiabilidad= .67; por ejemplo, "24.-Me aburro y me siento inquieto durante las vacaciones cuando no tengo nada productivo que hacer"), $D$ que está formado por 7 ítems (Fiabilidad $=.80$; por ejemplo, "3.-Me siento culpable cuando falto al trabajo") y $J$ formado por 10 ítems (Fiabilidad= .88; por ejemplo, "7.-Mi trabajo es tan interesante que a menudo no parece trabajo").

La escala de Irritación (Irritation Scale; Mohr, 1986; Mohr, Müller, Rigotti, Aycan y Tschan, 2006), en su versión en español (Merino et al., 2006). La presente escala tiene 8 ítems y 2 subescalas. La primera subescala se denomina "Irritación emocional" (fiabilidad $=.86)$ y está compuesta por 5 ítems (por ejemplo, "3.-Cuando otras personas se dirigen a mí, reacciono de malas maneras"); la segunda se llama "Irritación cognitiva" (fiabilidad $=.87$ ) y la integran 3 ítems" (por ejemplo, "1.-Me resulta difícil desconectar después del trabajo"). Las respuestas Likert se responden a través de 7 puntos (desde 1.-Muy en desacuerdo a 6.-Muy de acuerdo).

La escala de Burnout (MBI-GS; Schaufeli, Leiter, Maslach y Jackson, 1996), en la versión en lengua española (Salanova, Schaufeli, Llorens, Peiró y Grau, 2000), evalúa el burnout y consta de 15 ítems (3 subescalas). La subescala de "Agotamiento" comprende 5 ítems (por ejemplo, "6.-Estoy "quemado' por el trabajo"), la de "Cinismo" consta de 5 ítems (por ejemplo, "9.-He perdido entusiasmo por mi trabajo") y la de "Eficacia profesional" comprende 6 ítems (por ejemplo, "12.-He conseguido muchas cosas valiosas en este puesto"). El anclaje de respuestas es de 6 puntos (desde 1.ninguna vez a 7.-todos los días). La consistencia interna es de .87 (agotamiento), .85 (cinismo) y .78 (eficacia profesional).

El Inventario de Creencias Obsesivas (ICO; Belloch et al., 2003), en lengua española, evalúa las tendencias obsesivo-compulsivas. Consta de 58 ítems, agrupados en 7 factores que se contestan con una escala Likert de 7 puntos. Sólo se describen los dos factores utilizados en la investigación, que se refieren a los contenidos siguientes: 2.-Perfeccionismo e intolerancia a la incertidumbre (14 ítems; fiabilidad $=0,86$; por ejemplo, "2.- Debo ser el/la mejor en aquello que es importante para mí”) y 5.-Responsabilidad excesiva e importancia de controlar los pensamientos (10 ítems; fiabilidad= 0,84; por ejemplo, “49.- Debería ser capaz de librar mi mente de pensamientos inadecuados").

El Inventario de Impulsividad (DII; Dickman, 1990), en su versión española (Chico, Tous, Lorenzo-Seva y VigilColet, 2003), consta de 23 ítems y 2 subescalas. La primera evalúa la "impulsividad funcional" (fiabilidad $=.77 ; 11$ ítems; por ejemplo, "4.- Soy bueno aprovechando las ventajas de las oportunidades inesperadas, en las que tienes que hacer algo rápidamente o pierdes tu oportunidad") y la segunda subescala alude a la "impulsividad disfuncional" (fiabilidad $=.76 ; 12$ ítems; por ejemplo, "14.- Frecuentemente, me meto en situaciones apuradas porque no pienso antes de actuar'"). El formato de respuesta es dicotómico $(1=$ verdadero $/ 0=$ falso).

El Inventario de Personalidad (OPERAS; Vigil-Colet et al., en prensa) es un instrumento basado en el modelo de los cinco grandes factores de personalidad. La escala tiene un total de 40 ítems que se responden con una escala de 5 puntos. En cuanto a las propiedades psicométricas, los resultados muestran un buen ajuste del test a la estructura de 5 factores. Las características son: Extraversión (alfa $=.86$; por ejemplo, "2.-Soy el alma de la fiesta"), Estabilidad Emocional (alfa = .86; "32.-Cambio de humor a menudo"), Responsabilidad (alfa = .77; "5.-Siempre mantengo mi palabra"), Amabilidad (alfa = .71; “12.-Respeto a los demás") y Apertura a la Experiencia (al$f a=.81$; “24.-Me gusta probar cosas nuevas"). Además, en las puntuaciones obtenidas están corregidos los sesgos de deseabilidad social y de aquiescencia mediante la aplicación de procedimientos psicométricos específicos (Ferrando, Lorenzo-Seva y Chico, 2009; Lorenzo-Seva y Ferrando, 2009).

Por otro lado, también se utilizaron algunos correlatos (Del-Líbano et al., 2010) o también denominados indicadores externos (Gimeno, Benavides, Mira, Martínez y Benach, 2004) en forma de preguntas que los informantes debían de contestar. Algunas de ellas hacían referencia a "En términos generales, ¿te sientes saludable?", "Teniendo todo en cuenta la felicidad, ¿cómo estás de feliz con tu la vida?” y “¿Con qué frecuencia te llevas trabajo a casa?" y se contestaban a través de una escala Likert de cuatro anclajes (1.-Casi nunca, 2- $A$ veces, 3- $A$ menudo, 4-Casi siempre).

\section{Procedimiento}

Se utilizó el muestreo no probabilístico (Gómez, 1990; Hernández, Fernández y Baptista, 2000) o también denominado aleatorio-accidental (Kerlinger, 2001) para la obtención de las dos muestras. La tasa de respuesta fue del $85 \%$ (muestra 1) y del $81 \%$ (muestra 2). Se elaboró un protocolo que incluía una carta de presentación y un cuestionario que incluía todas las variables. Los participantes contestaron de forma voluntaria, no recibiendo ninguna gratificación ni monetaria ni en especies, en su lugar de trabajo habitual, previo consentimiento de los responsables de las empresas participantes. Un psicólogo experto estuvo presente durante la aplicación del cuestionario para resolver cualquier duda planteada por los participantes. La aplicación se realizó en un lugar tranquilo y con el tiempo suficiente para su adecuada cumplimentación. Se garantizó totalmente la confidencialidad de los datos aportados por los participantes.

\section{Análisis de Datos}

Se llevó a cabo un AFE (muestra 1) para estudiar la dimensionalidad de la prueba mediante el método de extracción de ejes principales y aplicando la rotación oblimin. Además, se utilizaron las matrices de correlación policórica ya que están especialmente indicadas en los casos en que los ítems presentan un formato de respuesta tipo Likert (Muthen y Kaplan, 1992). La utilización del programa FACTOR 7.2 (Lorenzo-Seva y Ferrando, 2006) para realizar el AFE es debido a que permite efectuar el análisis utilizando matrices de correlación policóricas. Además, proporciona diversos 
análisis, como por ejemplo el análisis paralelo, no disponibles en el SPSS 19.0. Por otro lado, éste último programa fue utilizado, en las dos muestras, tanto para evaluar la consistencia interna de las escalas (Alfa de Cronbach) como para calcular la validez convergente a través de la correlación establecida entre las dos subescalas del inventario que se presenta, con diversas variables de contraste (Tabla 3). Para la realización del AFC (muestra 2) se utilizó el modelo de ecuaciones estructurales denominado ESEM (Asparouhov y Muthén, 2009), se aplicó el Mplus (Versión 6.12). El modelo ESEM fue elegido ya que es apropiado para analizar medidas típicas y, además, es un modelo semirestringido que es idóneo para este tipo de datos y proporciona ajustes razonables (Ferrando y Lorenzo Seva, 2000; Hopwood y Donnellan, 2010).

\section{Resultados}

En la muestra 1, los resultados de la prueba de esfericidad de Bartlett (Chi-cuadrado, $d f 171=1666.5 ; p<0.01)$ y el índice Kaiser-Meyer-Olkin (KMO) de adecuación muestral de .846 mostraron la adecuación de los datos para la aplicación de un análisis factorial. El scree-test (Cattell, 1966) recomendó una solución de dos factores relacionados con $D$ y $J$. De forma complementaria, el análisis paralelo (Lattin, Carroll y Green, 2003; Timmerman y Lorenzo-Seva, 2011) y el criterio "minimum average partial" de Vellicer (1976) señalaron la adecuación de la solución de dos factores. La Figura 1 muestra el gráfico de sedimentación junto con el análisis paralelo indicando la solución bifactorial.

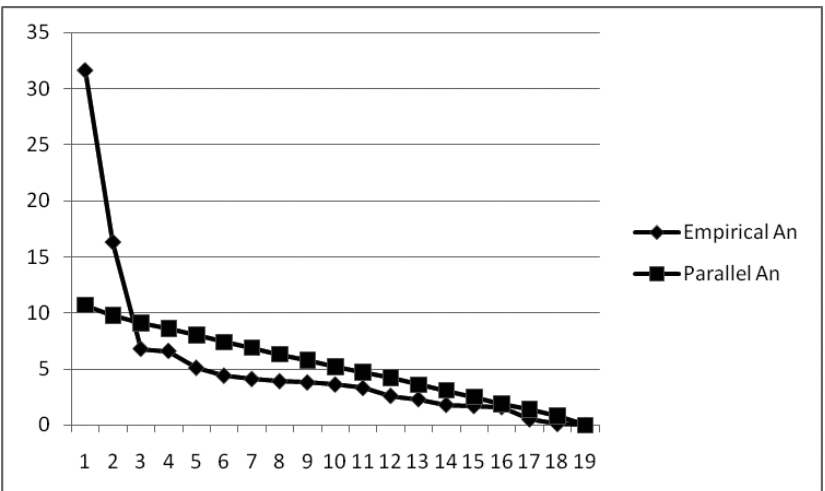

Figura 1.Gráfico de sedimentación de la escala WorkBAT de Spence y Robbins.

Una vez establecida la solución factorial más adecuada se utilizó el método de rotación Promin (Lorenzo-Seva, 1999) con el objetivo de obtener una solución factorial simple, dicho método de rotación oblicua tiende a obtener una solución lo más simple posible incluso en aquellos casos en que alguno de los ítems muestre una estructura compleja. A partir de los 25 ítems de la versión en inglés (Burke, 1999, 2001; McMillan et al., 2002; Spence y Robbins, 1992), se depuró la escala eliminando todos aquellos ítems que presentaran saturaciones inferiores a .30 o saturaciones complejas (superiores a .30 en más de un factor). De esta forma, se seleccionaron 19 ítems con las mayores saturaciones de tal modo que los dos factores están constituidos por 12 y 7 ítems respectivamente (Tabla 2).

Tabla 2. Matriz de saturaciones de los factores de la escala WorkBAT de Spence y Robbins de la solución bifactoriales (D y J) en lengua española.

\begin{tabular}{|c|c|c|c|}
\hline \multirow[t]{2}{*}{ Contenido Ítems } & \multirow[t]{2}{*}{ (a) } & \multicolumn{2}{|c|}{ (b) } \\
\hline & & F1-D & F2-J \\
\hline 2.-Me siento culpable cuando falto al trabajo.[3.-I feel guilty when I miss work] & $\mathrm{D}$ & .38 & -.01 \\
\hline $\begin{array}{l}\text { 4.-A menudo desearía no estar tan comprometido con mi trabajo.[5.-I often wish I wasn't so committed to my } \\
\text { work] }\end{array}$ & $\mathrm{D}$ & .39 & -.21 \\
\hline 8.-Perder el tiempo es tan malo como perder dinero.[12.-Wasting time is as bad as wasting money] & WI & .30 & .06 \\
\hline $\begin{array}{l}\text { 9.- Me siento obligado a trabajar duro incluso cuando no es agradable.[14.-I feel obliged to work hard even when } \\
\text { it is not pleasant] }\end{array}$ & $\mathrm{D}$ & .68 & -.17 \\
\hline $\begin{array}{l}\text { 10.-Pierdo la noción del tiempo cuando estoy involucrado en un proyecto.[16.-I lose track of time when I am in- } \\
\text { volved in a project] }\end{array}$ & $\mathrm{J}$ & .51 & .06 \\
\hline $\begin{array}{l}\text { 12.-Es importante para mí trabajar duro, aun cuando no me guste lo que estoy haciendo.[18.-It is important for } \\
\text { me to work hard, even if I am not enjoying what I am doing] }\end{array}$ & $\mathrm{D}$ & .75 & -.11 \\
\hline $\begin{array}{l}\text { 13.-Cuando me involucro en un proyecto interesante, es difícil describir cómo me siento de eufórico.[19.-It's dif- } \\
\text { ficult to describe how excited I feel when I get involved in an interesting project] }\end{array}$ & $\mathrm{J}$ & .51 & .13 \\
\hline $\begin{array}{l}\text { 14.-A menudo me encuentro pensando en el trabajo, aun cuando quiero descansar durante un tiempo.[20.-I of- } \\
\text { ten find myself thinking about work, even when I want to rest for a while] }\end{array}$ & $\mathrm{D}$ & .64 & -.02 \\
\hline $\begin{array}{l}\text { 15.-Entre mi trabajo y otras actividades en las que estoy implicado no dispongo de demasiado tiempo libre. [21.- } \\
\text { My work and the other activities I am involved in leave me with little free time] }\end{array}$ & WI & .50 & -.05 \\
\hline $\begin{array}{l}\text { 16.-A menudo siento que hay algo dentro de mí que me impulsa a trabajar duro.[22.-I often feel there is somet- } \\
\text { hing inside me pushing me to work hard] }\end{array}$ & $\mathrm{D}$ & .75 & 14 \\
\hline $\begin{array}{l}\text { 18.-Me aburro y me siento inquieto durante las vacaciones cuando no tengo nada productivo que hacer.[24.-I get } \\
\text { bored and get restless during holidays when I do not have anything productive to do] }\end{array}$ & WI & 41 & .11 \\
\hline $\begin{array}{l}\text { 19.-Me parece que tengo una compulsión interna a trabajar duro. [25.-It seems as if something inside me forces } \\
\text { me to work hard] }\end{array}$ & $\mathrm{D}$ & .71 & .15 \\
\hline 1.-Me gusta $n$ & J & .05 & .53 \\
\hline 3.-Mi trabajo es más diversión que trabajo.[4.-My job is more fur & $\mathrm{J}$ & -.07 & .83 \\
\hline
\end{tabular}


5.-Mi trabajo es tan interesante que a menudo no parece trabajo.[7.-My job is so interesting that it often doesn't $\quad$ J $\quad-.12$ seem like work]

6.-Trabajo más de lo que se espera de mí sólo para pasármelo bien. [9.-I work more than is expected of me just $\quad$ J $\quad .09 \quad \quad .58$

to enjoy myself]

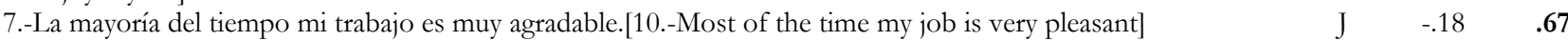

11.-A veces, cuando me levanto por la mañana, me muero de ganas de llegar al trabajo. [17.-Sometimes, when I $\quad$ J $\quad$.11 $\quad .59$ get up in the morning, I can't wait to get to work]

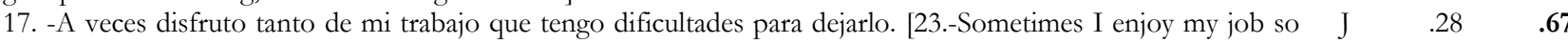
much that I find it difficult to stop working]

Cuando tengo tiempo libre, me gusta relajarme y no hacer cosas serias. [original inglés: 1.-When I have free time, WI

I like to relax and not do serious things]

Me gusta relajarme y divertirme todo lo que puedo. [original inglés: 6.-I like to relax and have fun as much as I WI

can]

Espero con ganas el fin de semana para pasármelo bien y no trabajar. [ original inglés: 8.-I really look forward to WI the weekend because I have fun and do not do any work]

Rara vez encuentro nada placentero mi trabajo. [ original inglés: 11.-I hardly ever find my job pleasant]

Paso mi tiempo libre ocupado en proyectos y otras actividades. [ original inglés: 13.-I spend my free time en- WI gaged in projects and other activities]

Me gusta usar mi tiempo de manera constructiva, dentro y fuera del trabajo. [ original inglés: 15.-I like to use my WI time constructively, whether I am working or not]

Varianza explicada (\%)

(a) Subescala original de Spence y Robbins (1992)

(b) Versión en lengua española $(\mathrm{N}=285)$.

(c) Ítem eliminado en la versión española.

La matriz de saturaciones de la solución factorial obtenida permite identificar los contenidos de los dos factores (Tabla 2), explicando entre todos ellos el $42.64 \%$ de la varianza. La correlación entre los dos factores es moderada 19 .

Dada la necesidad de confirmar la estructura del AFE se efectuó un AFC en la muestra 2. Los índices son: el error cuadrático medio de aproximación (RMSEA $\leq .06)$, el indice de ajuste comparado (CFI $\geq .95)$ y el indice de Tucker-Lewis (TLI $\geq$ .95). Los resultados obtenidos presentan un buen ajuste del modelo bifactorial dado que los índices (RMSE $A=.06 ; C F I$ $=.95$ y TLI $=.94)$ se sitúan cerca de los valores considerados óptimos. Los modelos de uno, tres o más factores han quedado descartados dado que no se ajustan.

En la Tabla 3 (muestras 1 y 2) se indican, la media, la desviación típica, los coeficientes de fiabilidad y los intervalos de confianza. También se presentan las correlaciones del instrumento que presentamos con variables demográficas (edad y antigüedad), con catorce correlatos y con cinco escalas (Irritación, MBI-GS, ICO, OPERAS y DII). Todo ello permite informar de los índices de validez del WorkBAT.

En este sentido, podemos observar cómo se establecen correlaciones entre los dos factores del WorkBAT y varios instrumentos (muestra 1). De esta forma, el factor que ex- presa motivación e implicación (D) correlaciona positivamente con ocho subescalas (por ejemplo, irritación emocional y cognitiva, agotamiento, eficacia personal, perfeccionismo, responsabilidad y impulsividad disfuncional) y el factor que hace alusión al disfrute en el trabajo (J) se asocia positivamente con cuatro (por ejemplo, eficacia personal, extraversión, estabilidad emocional e impulsividad funcional) $y$, también, correlaciona negativamente con dos (por ejemplo, cinismo y agotamiento). En cuanto a los correlatos externos utilizados las dos subescalas $(D$ y $J$ ) del WorkBAT se asocian a varios de ellos tanto directa como inversamente. Así, el factor $D$ se asocia con doce correlatos, diez de forma directa (por ejemplo, la frecuencia con que uno se lleva el trabajo a casa) y dos inversas (por ejemplo, la antigüedad en el lugar de trabajo actual). Por otro lado, el y el factor $J$ se correlaciona con once, ocho de forma directa (por ejemplo, las horas trabajadas durante la semana) y tres inversamente (por ejemplo, la antigüedad en la empresa). También en la muestra 2 se observan indicios de validez. Con el fin de determinar si existían diferencias significativas entre las correlaciones obtenidas en las dos muestras se ha aplicado la transformación z (al 1\%) de Fisher (Del-Líbano et al., 2010), en este sentido cabe destacar que no se ha apreciado ninguna diferencia significativa entre las dos muestras. 
Tabla 3. Estadísticos descriptivos, fiabilidad, intervalos de confianza, aspectos socio-demográficos, correlatos externos, correlaciones entre los factores D y J, y las escalas de contraste (Irritación, MBI-GS, ICO, OPERAS y DII) de las dos muestras.

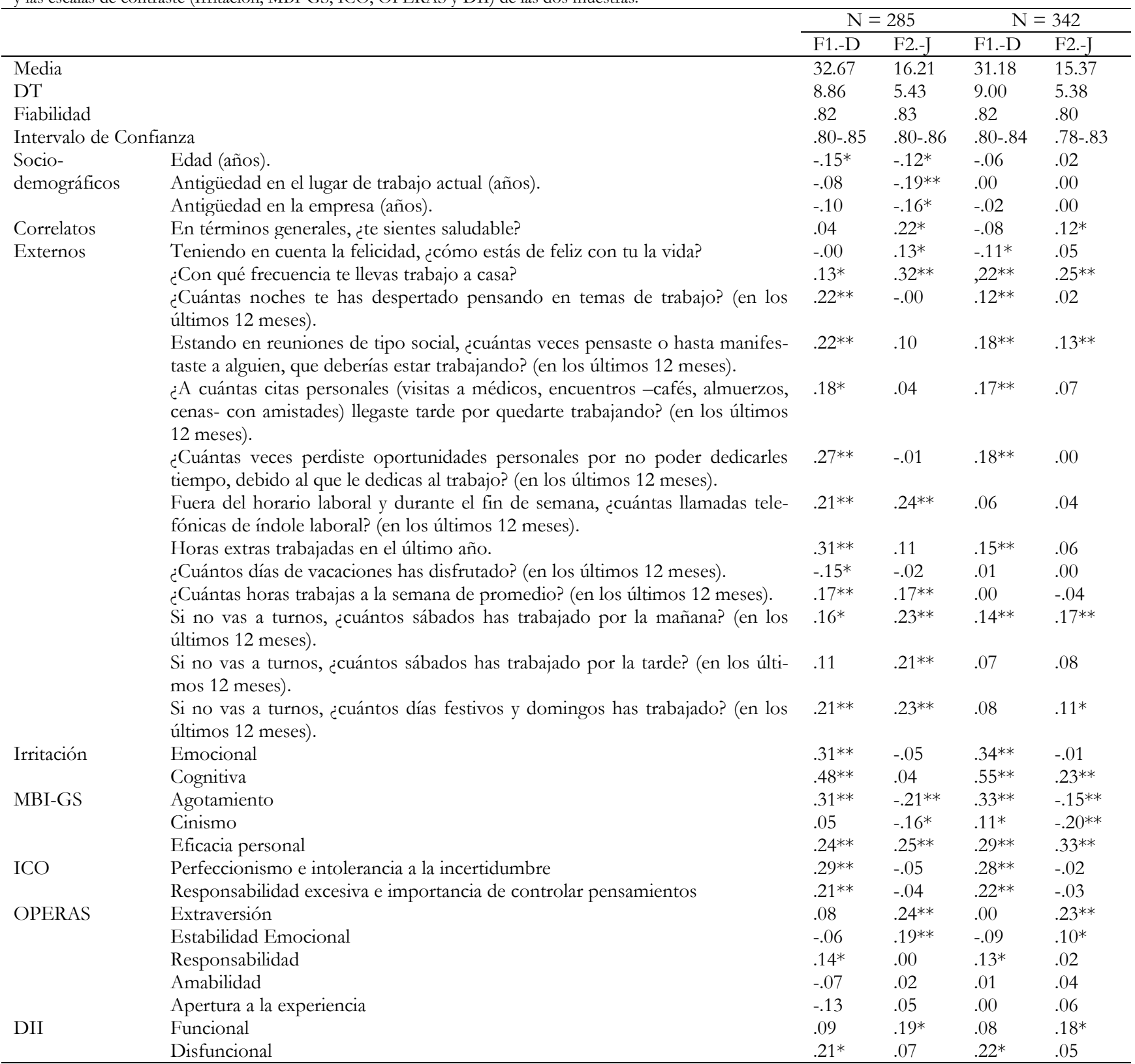

$* * p<.01 ; * p<.05$

\section{Discusión}

En el presente estudio presentamos las propiedades psicométricas de la escala WorkBAT de Spence y Robbins que consta de 19 ítems, es un instrumento que permite evaluar la adicción al trabajo a través de dos factores. Así, es la primera vez que dicha escala se presenta adaptada a la lengua española.

La primera hipótesis no se ha visto corroborada. Los resultados del AFE, con una muestra española heterogénea, no apoyan el modelo de tres factores que proponen algunos autores (Andreassen et al., 2010; Burke, 1999, 2001; Burke et al., 2002; Robinson, 1996; Spence y Robbins, 1992). No obstante, los resultados obtenidos en la presente investigación son congruentes con los encontrados en otras adaptaciones como las efectuadas por Kanai et al. (1996) (lengua japonesa), McMillan et al. (2002) (lengua inglesa, Nueva Zelanda), y Ersoy-Kart (2005) (lengua turca). Huang et al. (2010) por su parte proponen una solución pentafactorial, en lengua china, muy alejada de todas las anteriores.

$\mathrm{El}$ primer factor, el $D$ (Driven) (Alfa de Cronbach $=.82$ ), está relacionado con aquellos aspectos laborales que hacen 
referencia a la motivación, la involucración, la culpabilidad, el compromiso, la obligación, los pensamientos, la gestión del tiempo y del ocio, el impulso a trabajar, la compulsión y el aburrimiento cuando no se trabaja. El presente factor es muy similar a la versión de Ersoy-Kart (2005) que tiene once ítems. Es el que tiene más varianza explicando el $27.93 \%$ y está integrado por doce ítems (números: 2, 4, 8, 9, 10, 12, $13,14,15,16,18$ y 19) cabe destacar que en las otras versiones (Ersoy-Kart, 2005; Kanai et al., 1996; McMillan et al., 2002) se configura como el factor con menos varianza de los dos. Respecto al original de Spence y Robbins (1992), el presente factor está configurado por seis ítems de $D$, tres de $W I$ y dos ítems de $J$. Aunque sea de configuración mixta hemos respetado la denominación de $D$ por dos motivos, primero porque los ítems originales pertenecen mayoritariamente a este factor $\mathrm{y}$, segundo, porque las tres versiones bifactoriales (Ersoy-Kart, 2005; Kanai et al., 1996; McMillan et al., 2002) analizadas también lo hacen así.

El segundo factor, es $J$ (Work Enjoyment), hace referencia a las conductas de disfrute, de diversión, de pasarlo bien trabajando, de ser agradable y de "morirse de ganas de trabajar". Su fiabilidad es de .83. Está configurado por siete ítems (números 1, 3, 5, 6, 7, 11 y 17) que explican el 14.71\% de la varianza. Si lo comparamos con el original de Spence y Robbins (1992) se puede afirmar que los ítems coinciden totalmente respecto al factor de origen. Además, la configuración del presente factor en lengua española reproduce las versiones bifactoriales de Kanai et al. (1996), McMillan et al. (2002) y Ersoy-Kart (2005). Es de resaltar que en la solución bifactorial que presentamos, en español, hay seis ítems que han sido eliminados del original inglés (números: $1,6,8,11$, 13, 15). Además, tres ítems del original inglés (números: 1,6 y 13) son eliminados sistemáticamente por todas las soluciones bifactoriales propuestas en los distintos idiomas.

En cuanto a las correlaciones entre los dos factores ( $D$ y $J)$, en la versión española que presentamos es de .19, similar a la presentada por Ersoy-Kart (2005) de .20, y muy por debajo de las versiones inglesa de Nueva Zelanda (McMillan et al., 2002) y japonesa (Kanai et al., 1996) con .29 y .32 respectivamente.

La segunda hipótesis se cumple dado que el AFC apoya el modelo de los dos factores obtenido por el AFE. Todo ello se corrobora mediante los índices obtenidos dado que presentan un buen ajuste del modelo (RMSE $A=.06$; $C F I=$ .95 y $T L I=.94)$. Las saturaciones de los ítems oscilan entre .36 y .80 .

La tercera hipótesis se verifica ya que los coeficientes de fiabilidad obtenidos (en las dos muestras, ver tabla 3) en la versión española equivalentes a la versión turca. Los coeficientes hallados por Ersoy-Kart (2005) en las dos subescalas $(D$ y $J)$ son .83 . Para la solución bifactorial, los coeficientes de frabilidad de Kanai et al. (1996) para las dos escalas fueron aceptables (.70 para $D$ y .85 para $J)$. La investigación de McMillan et al. (2002) arroja que las fiabilidades fueron de .67 (D) y .84 (J) que son similares a la anterior. En la solución de cinco factores, los coeficientes oscilan entre .60 y .88
(Huang et al., 2010) y en la de tres factores (Andreassen et al., 2010; Burke, 2001) los intervalos están entre .63 y .67 (para WI), .80 y .82 (para $D)$, y .77 y .88 (para J).

La cuarta hipótesis era aportar indicios de validez del WorkBAT. En términos generales los datos resultantes del estudio (en las dos muestras) presentado indican que los factores de la escala analizada se asocian de forma significativa a otras escalas y a correlatos externos. El factor $D$ se asocia al perfeccionismo $(r=.29, p<.01)$, en nuestra versión, al igual que los resultados encontrados por Kanai et al. (1996). También, McMillan et al. (2002) encuentran que la compulsión y la obsesión se relacionan con $D$, éste extremo se confirma en la versión española $(r=.21, p<.01)$. Las investigaciones de Andreassen et al. (2007) muestran que las tres subescalas (agotamiento, cinismo y la eficacia personal) del burnout, evaluadas con el MBI-GS, presentan asociaciones. En la investigación que presentamos aparecen correlaciones similares con el MBI-GS: el agotamiento con $D(\mathrm{r}=.31, p$ $<.01)$ y con $J(\mathrm{r}=-.21, p<.01)$, el cinismo con $\mathrm{J}(r=-.16, p$ $<.05)$ y la eficacia personal con $D(r=.24, p<.01)$ y $J(r=.25, p$ $<.01)$.

Por otra parte, según Andreassen et al. (2010), los cinco factores de personalidad se asocian, a las subescalas del WorkBAT. La versión española que presentamos refuerza la vinculación de la adicción del trabajo, dado que se relaciona con extraversión con $J(r=.24, p<.01)$, estabilidad emocional con $J(r=.19, p<.01)$ y responsabilidad con $D(r=.14, p$ $<.05)$. En el mismo sentido, se puede indicar que tanto la irritación (cognitiva y emocional) como la impulsividad (funcional y disfuncional) presentan correlaciones significativas con los dos factores $(D$ y $J)$ del instrumento que presentamos.

Otros autores (Aziz y Zickar, 2006; Burke y Ng, 2007; Huang et al., 2010; Johnstone y Johnston, 2005; Porter, 1996) han utilizado correlatos externos como la edad y las horas trabajadas a la semana para determinar la validez del WorkBAT. En la presente investigación se confirma una correlación inversa de la edad para $D(r=-.15, p<.05)$ y para $J$ $(r=-.12, p<.05)$ y las horas trabajadas durante la semana se relacionan con $D$ y $J(r=.17 ; p<.01)$. Además la versión española (factores $D$ y $J$ ) la hemos asociado a otros índices con los cuales se han obtenido correlaciones significativas como la antigüedad en el lugar de trabajo actual (con $J, r=-.19, p$ $<.01$ ) y en la empresa (con $J, r=-.16, p<.05$ ), las noches que uno se ha despertado pensando en temas de trabajo (con $D, r=.22, p<.01$ ), las reuniones de tipo social (con $D$, $r=.22, p<.01$ ), las citas personales impuntuales (con $D, r=$ $.18, p<.05$ ), la pérdida de oportunidades personales (con $D, r=.27, p<.01$ ), las llamadas telefónicas de carácter laboral fuera del horario laboral o en fines de semana (con $D, r=$ $.21, p<.01$ y con $\mathrm{J}, r=.24, p<.01$ ), las horas extras trabajadas en el año (con $D, r=.31, p<.01)$, los días de vacaciones disfrutados al año (con $D, r=-.15, p<.05)$, si no se va a turnos los sábados trabajados por la mañana (con $D, r=.16$, $\mathrm{p}<.05$ y con $J, r=.23, p<.01)$ y por la $\operatorname{tarde}(\operatorname{con} J, r=.21$, 
$p<.01)$, y los festivos y domingos trabajados si no se va a turnos ( $\operatorname{con} D, r=.21, p<.01$ y con $J, r=.23, p<.01)$.

En conclusión, teniendo en cuenta los resultados de la presente investigación, podemos indicar que la escala WorkBAT presenta unas aceptables propiedades psicométricas y permite determinar el comportamiento de la adicción al trabajo. En primer lugar, la versión española del WorkBAT se configura como bifactorial y es muy similar a las halladas por Kanai et al. (1996), McMillan et al. (2002) y Ersoy-Kart (2005); además se reduce el número de ítems de los veinticinco originales a los diecinueve. Por otro lado, los coeficientes de fiabilidad de los dos factores son adecuados y son semejantes a la versión en lengua turca (Ersoy-Kart, 2005). $\mathrm{Y}$ tercero, hemos encontrado indicadores externos de la adicción al trabajo confirmando que pueden ser utilizados para determinar la validez de la escala.

Consideramos que la presente investigación contribuye al conocimiento de la adicción al trabajo en varios aspectos. Los resultados proporcionan importantes implicaciones prácticas con respecto a la adicción al trabajo que deberían de considerarse para una adecuada gestión estratégica de los recursos humanos (Boada-Grau y Gil-Ripoll, 2010, 2011) dentro de las organizaciones. En primer lugar, se requerirán investigaciones adicionales para examinar el proceso evolutivo por las cuales un adicto al trabajo puede ir transitando en el proceso de adicción (Chamberlin y Zhang, 2009), es más, sería necesario identificar cómo la adicción al trabajo afecta a las personas en las diferentes etapas que configuran su ciclo vital y profesional. Segundo, también será preciso analizar muestras de distintas culturas con el fin de explorar si la adicción al trabajo es un constructo universal, así pues debería de escudriñarse la relación entre la adicción al trabajo, los valores culturales y la ética del trabajo y, de forma singular, la ética protestante del trabajo (Porter, 1996). Y finalmente, la escala WorkBAT puede ser empleada como una herramienta para el diseño de intervenciones dirigidas aumentar la calidad de vida laboral de los trabajadores. Los ti-

\section{Referencias}

Andreassen, C. S., Hetland, J. y Pallesen, S. (2010). The relationship between 'workaholism', basic needs satisfaction at work and personality. European Journal of Personality, 24, 3-17.

Andreassen, C. S., Ursin, H. y Eriksen, H. R (2007). The relationship between strong motivation to work, "workaholism", and health. Psychology y Health, 22, 615-629.

Asparouhov, T. y Muthén, B. (2009). Exploratory structural equation modeling. Structural Equation Modeling, 16, 397-438.

Aziz, S. y Zickar, M. J. (2006). A cluster analysis investigation of workaholism as a syndrome. Journal of Occupational Health Psychology, 11, 52-62.

Bakker, A, B., Demerouti, E. y Burke, R. (2009).Workaholism and Relationship Quality: A Spillover-Crossover Perspective. Journal of Occupational Health Psychology, 14, 23-33.

Belloch, A., Cabedo, E., Morillo, C., Lucero, M. y Carrió, C. (2003). Diseño de un instrumento para evaluar las creencias disfuncionales del trastorno obsesivo-compulsivo: resultados preliminares del Inventario de Creencias Obsesivas (ICO). International Journal of Clinical and Health Psychology, 3, 235-250.

Boada-Grau, J. (1999). Psicología del (Trabajo +Organizaciones + Recursos Humanos). Barcelona: PPU Ediciones. pos de intervenciones (Boada-Grau, 1999) donde podría ser utilizada la presente escala son la primaria (dirigida a eliminar las conductas adictivas al trabajo), la secundaria (que persigue reducir la incidencia y prevalencia de las conductas adictivas) y la terciaria (orientada a paliar los efectos negativos y la destructividad derivada de las conductas adictivas). Un ejemplo de intervención psicosocial serían los Programas de Ayuda al Empleado (Solé y Balduque, 2006) los cuales pueden facilitar información valiosa para evitar las conductas adictivas al trabajo tanto presentes como futuras.

Las limitaciones del presente estudio son el origen de las investigaciones que pretendemos desplegar en el futuro, éstas las comentamos a continuación. Primera, sería preciso analizar la validez discriminante (Boada-Grau, González, Vigil-Colet, Mañas y Agulló, 2009; Boada-Grau, SánchezGarcía, Prizmic-Kuzmica y Vigil-Colet, 2012) del inventario tanto entre colectivos de trabajadores y profesionales de origen diverso que sean competentes en lengua española (por ejemplo, africano, latinoamericano y centroeuropeo) como atendiendo al tipo de actividad profesional ("cuello blanco" vs "cuello azul"). Segunda, es probable que nuevas investigaciones aporten nuevos datos sobre la validación de una escala ya que esta es un proceso dinámico que no finaliza con la construcción y publicación del mismo (Padilla, Gómez, Hidalgo y Muñiz, 2006). Tercera, en investigaciones futuras deberían de incluirse las evaluaciones que los compañeros de trabajo y/o los supervisores (Andreassen et al., 2010) pueden realizar sobre un empleado. Y por último, deberían de utilizarse estudios longitudinales con el fin de determinar las consecuencias de la adicción al trabajo y, además, la relación entre ésta y otras variables como la formación, el crecimiento personal, la promoción profesional y los conflictos trabajo vs familia (Huang et al., 2010).

Agradecimientos.- Esta investigación ha sido realizada gracias a la subvención recibida del Ministerio de Ciencia e Innovación (MICINN), proyecto con la referencia DER2010-21686-C02-02.

Boada-Grau, J. y Gil-Ripoll, C. (2010). Strategic human resources management as an antecedent to the Balanced Scorecard. Psychology in Spain, 14, 64-73.

Boada-Grau, J. y Gil-Ripoll, C. (2011). Measure of human resource management practices: psychometric properties and factorial structure of the questionnaire PRH-33. Anales de Psicología, 27, 527-535.

Boada-Grau, J., González, S., Vigil-Colet, A., Mañas, M. A. y Agulló, S. (2009). CONS-32: Development of a laboral risk prevention questionnaire for the construction industry. Psicothema, 21, 165-169.

Boada-Grau, J., Sánchez-García, J. C., Prizmic-Kuzmica, A-J. y Vigil-Colet, A. (2012). Work health and hygiene in the transport industry (TRANS18): Factorial structure, reliability and validity. The Spanish Journal of Psychology, 15, 357-366.

Bonebright, C. A., Clav, D. L. y Ankenmann, R. D. (2000). The relationship of workaholism with work-life conflict, life satisfaction, and purpose in life. Journal of Counseling Psychology, 47, 469-477.

Brislin, R.W. (1970). Back-translation for cross-cultural research. Journal of Cross-Cultural Psychology, 1, 185-216.

Burke, R. J. (2000). Workaholism in organizations: psychological and physical well-being consequences. Stress Medicine, 16, 11-16. 
Burke, R. J. (2001). Spence and Robbins' measures of workaholism components: test-retest stability. Psychological Reports, 88, 882-888.

Burke, R. J., Matthiesen, S. B. y Pallesen, S. (2006). Personality correlates of workaholism. Personality and Individual Differences, 40, 1223-1233.

Burke, R. J., Richardsen, A. M. y Martinussen, M. (2002). Psychometric properties of Spence and Robbins' measures of workaholism components. Psychological Reports, 91, 1098-1104.

Burke, R. J. (1999). Workaholism in organizations: measurement validation and replication. International Journal of Stress Management, 6, 45-55.

Burke, R. J. y Ng, E. S. W. (2007). Workaholic Behaviors: Do Colleagues Agree?. International Journal of Stress Management, 14, 312-320.

Cattell, R. B. (1966). The scree test for the number of factors. Multivariate Behavioral Research, 1, 245-276.

Chamberlin, C. M. y Zhang, N. (2009). Workaholism, Health, and SelfAcceptance. Journal of Counseling y Development, 87, 159-169.

Chico, E., Tous, J.M., Lorenzo-Seva, U. y Vigil-Colet, A. (2003). Spanish adaptation of Dickman's impulsivity inventory: its relationship to Eysenck's personality questionnaire. Personality and Individual Differences, 35, 1883-1892.

Del-Líbano, M., Llorens, S., Salanova, M. y Schaufeli, W. (2010). Validity of a brief workaholism scale. Psicothema, 22,143-150.

Dickman, S. J. (1990). Functional and dysfunctional impulsivity: personality and cognitive correlates. Journal of Personality and Social Personality, 58, 95102.

Ersoy-Kart, M. (2005). Reliability and validity of the workaholism battery (Work-BAT): Turkish form. Social Behavior and Personality, 33, 609-618.

Ferrando, P. J. y Lorenzo-Seva, U. (2000). Unrestricted versus restricted factor analysis of multidimensional test items: some aspects of the problem and some suggestions. Psicológica, 21, 301-323.

Ferrando, P.J., Lorenzo-Seva, U. y Chico, E. (2009). A General FactorAnalytic Procedure for Assessing Response Bias in Questionnaire Measures. Structural Equation Modeling-a Multidisciplinary Journal, 16, 364 381.

Gimeno, D., Benavides, F. G., Mira, M., Martínez, J. M. y Benach, J. (2004). External validation of psychological job demands in a bus driver sample. Journal of Occupational Health, 46, 43-48.

Gómez, J. (1990). Metodología de encuesta por muestreo. En J. Arnau, M. T. Anguera y J. Gómez (Dirs.). Metodología de la Investigación en Ciencias del Comportamiento (pp.239-310). Murcia: Universidad de Murcia.

Hambleton, R. K. (1994). Guidelines for adapting educational and psychological tests: A progress report. European Journal of Psychological Assessment, 10, 229-244.

Hambleton, R. K., Merenda, P. F. y Spielberger, C. D. (2005). Adapting educational and psychological tests for cross-cultural assessment. London: Lawrence Erlbaum Associates.

Haymon, S. W. (1992). The relationship of work addiction and depression, anxiety, and anger in college males (Doctoral dissertation, Florida State University, 1992). Dissertation Abstracts International, 53, 5401B.

Hernández, R., Fernández, C. y Baptista, P. (2000). Metodología de la investigación. México: McGraw-Hill.

Hopwood, C. J. y Donnellan, M. B. (2010). How should the internal structure of personality inventories be evaluated? Personality and Social Psychology Review, 14, 332-346.

Huang, J-C., Hu, C. y Wu, T-C. (2010). Psychometric properties of the chinese version of the workaholism battery. The Journal of Psychology, 144, 163-183.

Jackson, D. L. (1992). Correlates of physical and emotional health among male and female workaholics (Doctoral dissertation, University of Oregon, 1993). Dissertation Abstracts International, 53 (10), 5446.

Johnstone, A. y Johnston, L. (2005). The relationship between organizational climate, occupational type and workaholism. New Zealand Journal of Psychology, 34, 181-188.

Kanai, A. y Wakabayashi, M. (2001). Workaholism among Japanese bluecollar employees. International Journal of Stress Management, 8, 129-145.

Kanai, A., Wakabayashi, M. y Fling, S. (1996). Workaholism among employees in Japanese corporations: An examination based on the Japanese version of the workaholism scales. Japanese Psychological Research, 38 , 192-203.

Kerlinger, F. N. (2001). Investigación del comportamiento: Métodos de investigación en ciencias sociales. México: McGraw-Hill.
Lattin, J., Carroll, D. J. y Green, P. E. (2003). Analyżing multivariate data. Pacific Grove: Duxbury Press.

Lorenzo-Seva, U. (1999). Promin: a method for oblique factor rotation. Multivariate Behavioral Research, 34, 347-365.

Lorenzo-Seva, U. y Ferrando, P. J. (2006). FACTOR: A computer program to fit the exploratory factor analysis model. Behavioral Research Methods, Instruments and Computers, 38, 88-91.

Lorenzo-Seva, U. y Ferrando, P.J. (2009). Acquiescent responding in partially balanced multidimensional scales. British Journal of Mathematical y Statistical Psychology, 62, 319-326.

Maslach, C. (1982). Burnout - The cost of caring. Englewood Cliffs, NJ: Prentice Hall.

Maslach, C., Jackson, S. E. y Leiter, M. P. (1996). Maslach burnout inventor manual. Palo Alto, CA: Consulting Psychologists Press, Inc.

McMillan, L. H. W., O'Driscoll, M. P, Marsh, N. V. y Brady, E. C. (2001) Understanding workaholism: Data synthesis, theoretical critique, and future design strategies. International Journal of Stress Management, 8, 69-91.

McMillan, L. H. W., Brady, E.C., O'Driscoll, M. P. y Marsh, N. V. (2002). A multifaceted validation study of Spence and Robbins' (1992) Workaholism Battery. Journal of Occupational and Organizational Psychology, 75, $357-$ 368.

Merino, E., Carbonero, M. A., Moreno, B. y Morante, M.E. (2006). Irritation: Analysis of an instrument to assess stress at work. Psicothema, 18, 419-424.

Mohr, G. (1986). Die erfassung psychischer befindensbeeinträchtigungen bei Industriearbeitern. Europäische hochschulschriften. Frankfurt: Lang.

Mohr, G., Müller, A., Rigotti, T., Aycan, Z. y Tschan, F. (2006). The assessment of psychological strain in work contexts: concerning the structural equivalency of nine language adaptations of the Irritation-scale. European Journal of Psychological Assessment, 22, 198-206.

Muñiz, J. y Bartram, D. (2007). Improving international tests and testing. European Psychologist, 12, 206-219.

Muthen, B. y Kaplan, D. (1992). A comparison of some methodologies for the factor analysis of non-normal Likert variables: A note on the size of the model. British Journal of Mathematical and Statistical Psychology, 45, 1930

Ng, T. W. H., Sorensen, K. L. y Feldman, D. C. (2007). Dimensions, antecedents, and consequences of workaholism: A conceptual integration and extension. Journal of Organizational Behavior, 28, 111-136.

Oates, W. E. (1968). On being a 'workaholic' (a serious jest). Pastoral Psychology, 19, 16-20

Oates, W.E. (1971). Confessions of a workabolic. New York: World.

Padilla, J. L., Gómez, J., Hidalgo, M. D. y Muñiz, J. (2006). Evaluation of the consequences of using tests on validity theory. Psicothema, 18, 307-312.

Poelmans, S. Y. y Masuda, A. D. (2009). Work Addiction. In Workplace Psychological Health: Current Research and Practice. En P. Brough, M. O'Driscoll, T. Kalliath, C. L. Cooper y S. Y. Poelmans (Editors). Workplace Psychological Health: Current Research and Practice (pp. 87-97). Cheltenham, UK: Edward Elgar Publishing.

Porter, G. (1996). Organizational impact of workaholism: Suggestions for researching the negative outcomes of excessive work. Journal of Occupational Health Psychology, 1, 70-84.

Robinson, B. E. (1998). Chained to the desk: A guidebook for workabolics, their partners and children and the clinicians who treat them. New York: NYU Press.

Robinson, B. E. (1996). Current validity of the work addiction risk test as a measure of workaholism. Psychological Reports, 79, 1313-1314.

Robinson, B. E. (2000). Workaholism: Bridging the gap between workplace, sociocultural, and family research. Journal of Employment Counseling, 37, $31-47$

Salanova, M., Schaufeli, W.B., Llorens, S., Peiró, J.M. y Grau, R. (2000). Desde el 'burnout' al 'engagement': ¿una nueva perspectiva? Revista de Psicología del Trabajo y las Organizaciones, 16, 117-134.

Schaufeli, W. B., Taris, T. W. y Rhenen, W. V. (2008). Workaholism, burnout, and work engagement: Three of a kind or three different kinds of employee well-being? Applied Psychology: International Review, 57, 173-203.

Schaufeli, W. B., Leiter, M. P., Maslach, C. y Jackson, S. E. (1996). Maslach Burnout Inventory - General Survey. En C. Maslach, S. E. Jackson y M. P. Leiter (Eds.). The Maslach Burnout Inventory- Test Manual (3rd ed.) Palo Alto, CA: Consulting Psychologists Press. 
Schaufeli, W. B., Shimazu, A. y Taris, T.W. (2009). Driven to work excessively hard: The evaluation of a two-factor measure of workaholism in the Netherlands and Japan. Cross-Cultural Research, 43, 320-348.

Scott, K. S., Moore, K. S. y Miceli, M. P. (1997). An exploration of the meaning and consequences of workaholism. Human Relations, 50, 287314.

Solé, M. D. y Balduque, M. (2006). El programa de ayuda al empleado (EAP): intervención individual en la prevención de riesgos psicosociales. Madrid: Instituto Nacional de Seguridad e Higiene en el Trabajo.

Spence, J. T. y Robbins, A. S. (1992). Workaholism: Definition, measurement, and preliminary results. Journal of Personality Assessment, 58, 160178.
Timmerman, M. E. y Lorenzo-Seva, U. (2011). Dimensionality assessment of ordered polytomous items with parallel analysis. Psychological Methods, 16, 209-220.

Vellicer, W. F. (1976). Determining the number of components from the matrix of partial correlations. Psychometrika, 41, 321-327.

Vigil-Colet, A., Morales-Vives, F., Camps, E., Tous, J. y Lorenzo-Seva, U. (en prensa) Development and validation of the Overall Personality Assessment Scale (OPERAS). Psicothema.

Whiteside, S. P. y Lynam D. R. (2001).The Five Factor Model and impulsivity: using a structural model of personality to understand impulsivity. Personality and Individual Differences, 30, 669-689.

(Artículo recibido: 3-3-2012; revisado: 28-8-2012; aceptado: 13-9-2012) 